\title{
Landscape Design Method of Urban Wetland Park Using the Building Information Model
}

\author{
Jiye $\mathrm{He}$ iD $^{1,2}$ \\ ${ }^{1}$ Guangdong University of Science \& Technology, Dongguan 523083, Guangdong, China \\ ${ }^{2}$ University of Perpetual Help System DALTA, Manila 1740, Philippines \\ Correspondence should be addressed to Jiye He; hejiye@gdust.edu.cn
}

Received 31 December 2021; Revised 18 January 2022; Accepted 19 January 2022; Published 16 February 2022

Academic Editor: Xin Ning

Copyright (c) 2022 Jiye He. This is an open access article distributed under the Creative Commons Attribution License, which permits unrestricted use, distribution, and reproduction in any medium, provided the original work is properly cited.

Wetland has long been referred to as the "Kidney of the Earth" because of its many functions, including water conservation, flood control, sewage treatment, and biodiversity conservation. Urban wetland parks are important for maintaining urban ecological processes and improving human settlement health and safety. The construction of urban wetland parks in my country has a certain gap with that of other countries in terms of theoretical research, management and operation, and construction experience. However, as a unique ecological system, wetlands are experiencing unprecedented destruction as a result of the current social economy's rapid development and the blind and disorderly expansion of human activities, and their area is shrinking or even disappearing. The current BIM policy has progressed from an initial application promotion and guidance stage to a comprehensive promotion and multipolicy integration development stage. BIM will be further integrated with related policies in the future, such as prefabricated buildings and the internet, as well as integrated management of service construction projects. Scientific decision-making, as well as improvements in quality and efficiency, will all contribute to the construction industry's healthy development.

\section{Introduction}

Wetland ecosystems have unique functions and benefits, and they are one of the most diverse ecological perspectives and important living environments for humans. However, as a large population center, the city's ecological environment is unquestionably concerning [1]. Severe phenomena and situations, such as the rapidly growing population, the rapidly developing economic situation, and the expanding urban scope, have resulted in a slew of ecological security issues, including shrinking wetland areas and overexploitation of wetland resources. Urban development, among the human activities that cause habitat loss, contributes the most to the extinction rate of local species and frequently results in the extinction of most local species [2]. Cities' "kidneys" are urban wetlands, and protecting and constructing wetlands is an important part of urban ecological construction. The construction of an urban wetland park contributes to the healthy development of the urban wetland natural ecological environment as well as the creation of a pleasant urban living environment. The most significant distinction between urban wetlands and other natural wetlands is that they are found in or adjacent to cities and are heavily influenced by the urban environment, economic development, and social and cultural forms [3]. People's perceptions of urban wetland park are somewhat skewed, which not only fails to fully appreciate the park's positive attributes but also contributes to its low level of landscape construction. The great change in urban landscape is the most visible external manifestation of the urbanization process and social and economic development. At the same time, it results in the loss of wetland landscape characteristics and a large-scale reduction of urban river wetland. In fact, many cities have lost a large portion of their wetlands. China is a large wetland country, with the fourth largest wetland area in the world, but wetland is one of the components of urbanization that disappears the fastest. As a result, protecting and studying urban wetland parks are critical [4]. 
The residents living in cities have been far away from nature. They are tired, disgusted, and even disappointed with the high-rise buildings of reinforced concrete, thick "cement nails," and gray city sky, and they yearn for "garden" cities more and more strongly. Urban wetland park is based on abundant wetland natural ecological resources and good wetland landscape resources [5]. With the functions of popular science education, leisure tourism, and cultural communication as the supplement, it provides an important popular science education place for people to know and understand the wetland ecosystem, which is helpful to raise people's awareness of scientific environmental protection. At the same time, the construction of urban wetland parks has a certain scale of service facilities, which makes them a good place for people to relax and visit [6]. Water and land on the Earth interact to form wetlands, so their ecological functions are extremely important. At the same time, wetlands also contain extremely rich biological resources, which can be protected by adjusting ecological links [7]. In the construction of urban wetland park landscape, the ecology of the wetland natural environment is the main factor. The landscape design should meet the harmonious relationship between nature and man-made. The key factor for the success of its construction is the formation of a virtuous cycle of wetland ecosystem. The construction of urban wetland park landscape provides favorable conditions for ecological protection, popular science education, natural wild interest, and leisure tourism. At the same time, it also realizes the sustainable development of wetland resources and the vision of harmonious coexistence between man and nature [8]. As an important natural carrier in the city, the landscape design of urban wetland park to properly deal with the relationship between tourists' leisure and wetland natural ecological environment is of practical significance in protecting the wetland ecosystem, building livable living environment, and realizing the sustainable development of urban wetland park [9]. Although there has been extensive research on wetland protection in developed countries abroad, research on the construction practice of urban wetland parks is still in its infancy. Wetland parks have been promoted as a model of rational utilization of urban wetlands in just a few years, and scientific research on wetlands, research on protection and rational utilization, and research on restoration and reconstruction around wetland parks have all advanced significantly [10]. Cities, like all living things in nature, are organic aggregates with an internal order that is similar to that of living organisms. The growth law of organisms governs the spread and development of cities. When part of the order in an organism is disrupted, the organism as a whole becomes paralyzed and dies. The biological evolution with nature can be used to deduce the principle of urban development. The importance of studying the social impact of urban information construction is elaborated in detail in this paper, focusing on urban wetland parks, creating diversified modern urban parks with the functions of ecology, science popularization, education, and landscape, promoting self-improvement and development, and raising public awareness of environmental protection [11].
Although urban wetland park plays an important role in protecting urban wetland park, the construction of urban wetland park in China is still in the primary stage compared with foreign countries, and the relevant theoretical research is not perfect; especially, the ecological concept, method, technology $w$, and its application in urban wetland park lack systematic theoretical research and practical experience [12]. At present, due to the unclear classification system, imperfect subjective evaluation standards, and many approval departments, urban wetland parks have problems such as blind construction, mixed types, and cumbersome management in the formulation and implementation process of standards [13]. With the extensive development of wetland protection and rational utilization of wetland resources, the development of wetland protection in China has reached a brand-new milestone. People's attention is no longer focused solely on wetland protection itself, but more and more on related aspects such as wetland protection and urban development. Therefore, urban wetland parks are gradually coming into everyone's vision [14]. This paper also has a deeper understanding of the urban wetland park landscape and landscape architecture through the tourists, reasons, and frequency of the tour. Based on the relationship between the urban information model and the natural environment of the wetland, the causes of the urban wetland park landscape and the ways of infiltration are understood, and some design methods for the present urban information architecture by using the reinforced block masonry building structure are also put forward [15].

\section{Related Work}

With the rapid development of social economy, the rhythm of people's life is constantly accelerating, and the pressure of life is also increasing. Some masters with keen insight apply organic concepts to the theories and applications in urban planning, architectural design, landscape ecology, and other fields. Hu [16] put forward organic architecture, which advocates that teachers should learn from the natural design concept and draw inspiration from the organic nature. The principles of urban development can be derived from the biological evolution of nature, and architecture should live in harmony with nature. When [17] mentions wetlands and swamps, it will first take into account the sharp contrast between the concepts of drainage and reconstruction. There are four design tendencies, natural design, rural design, protective design, and restorative design, which make more and more urban wetland parks designed and built. In [18], from the perspective of the concept of sustainable development, according to the principles of wetland landscape planning and design, the macroconcept of sustainable development is refined and applied to the planning and design of urban wetland parks, and relevant strategies for planning and design of urban wetland parks are put forward to grasp the direction of planning and design of urban wetland parks. In [19], project cost knowledge data are linked to BIM technology, combining the intelligence of the cost knowledge database with the parameterized and digitized characteristics of BIM technology to protect the wetland 
ecological environment, display wetland landscape characteristics, and improve urban ecological environment construction. According to [20], BIM has been used in businesses to some extent, but more work is needed to accelerate the adoption of the BIM, collaborative network offices, and other emerging industries in the construction market, as well as promote the adoption of information standards. In [21], we can give full play to its scientific and educational functions in urban wetland parks and design popular science activities based on the basic situation of aquatic plants' and animals' activities in different seasons in order to increase public awareness of wetlands and improve the ecosystem. By combining RS and GIS with dynamic roughness length (RL) of the underlying surface and field of view factor (SVF) of the surrounding sky, Zhang [22] proposed a reasonable layout of urban space, enhancement of urban permeability and microcirculation ability, and evaluation of ventilation potential. In [23], according to the discussion on housing and urban construction, the application level of information technology can be continuously improved, and the technical progress and management level of the construction industry can be promoted through overall planning and policy guidance, as well as further strengthening the information management of construction enterprises. In [24], in the case of wetlands, direct management of nature according to local conditions is used to achieve harmonious coexistence between man and nature, and the wetland environment and popular science wetland culture are displayed in the overall planning. Dai and $\mathrm{Xu}$ [25] pointed out that the compilation method and index system of regulation and control of urban wetland parks in China are not in-depth standardized and lack scientific and technological basis, which seriously affects the healthy development of urban wetland parks, which shows that the three key words of pharmaceutical city, wetland, and park are to understand the positioning and build urban wetland parks.

The above research shows that BIM has begun to invest in-depth research in the efforts of major industrial parks and relevant personnel, sort out some landscape status quo in combination with the integration of human and nature, determine the starting point and foothold, and summarize and apply them to the landscape, so as to meet the ecological design methods, form, and materials. Function and other aspects meet the ecological optimization of urban wetland landscape design and elastic development and maintain its developability. This paper will also conduct in-depth research and analysis in combination with the above relevant theories to promote ecological development.

\section{Materials and Methods}

3.1. Related Concepts of the Urban Information Model. In today's society, with the continuous improvement of the level of information technology $[26,27]$ and the widespread use of information technology, the global industry is triggering an information revolution. After realizing that information technology has brought huge benefits to the manufacturing industry, the construction industry began to pay attention to the application of information technology, and then BIM technology was born. In recent years, BIM has been applied in scientific research, universities, enterprises, governments, etc., such as the construction of the Shanghai Tower and the construction of the Hong Kong-ZhuhaiMacao Bridge, and has received unanimous praise from the society. The classification of the BIM city information model is shown in Figure 1.

It is a digital expression of the entity and functional characteristics of engineering project facilities, and it is a product information model based on three-dimensional digital technology, integrating various related information of construction projects. To some extent, the emergence of BIM information technology has realized information sharing in the entire cycle from construction to operation and maintenance for related construction enterprises, which is conducive to the visualization, predictability, and controllability of related technologies for related construction enterprises over time [28]. It is the formal expression of physical and functional characteristics of building facilities in accordance with open industrial standards, as well as other computable or operational information pertaining to the entire construction project life cycle. BIM [29] organizes all relevant information in a continuous application program, allowing users to extract, change, and perform other operations. By drawing on paper, BIM architecture can give more priority to project planning and turn to real-time data sharing. Transparency, writing, control, and, ultimately, better structure can all be achieved through this process. It can better predict how long a project will take, which helps to cut down on labor costs. At the same time, it can save our previous data, delete and discard some errors, and leave us with useful information. It has no space limitations and is unquestionably the best option for urban wetland parks. The following is a summary of the BIM model construction cost formula:

total cost $=\sum$ [quantities $\times$ unit price $\times$ consumption index $]$.

Related concepts of the urban information model (BIM) are one of the most important protection means for wetland parks. It can unify the construction and management of wetland park's structure, characteristics, landscape, ecology, and other processes in the natural wetland ecosystem and emphasize the unity of protection and utilization [30]. Various information means are constantly applied to people's construction, production, and daily lives, which not only effectively improves the work efficiency of production and life but also saves a significant amount of labor and provides a great deal of convenience to people. Each wall must be hoisted reasonably during the assembly construction of the building structure. Reasonable tower crane use can not only maximize tower crane utilization and reduce idling as much as possible but can also effectively reduce the cost of mechanical shift in the construction process. When a certain lifting torque is applied, the lifting radius decreases as the weight of the wall to be assembled rises. The general crane lifting moment curve equation is as follows: 


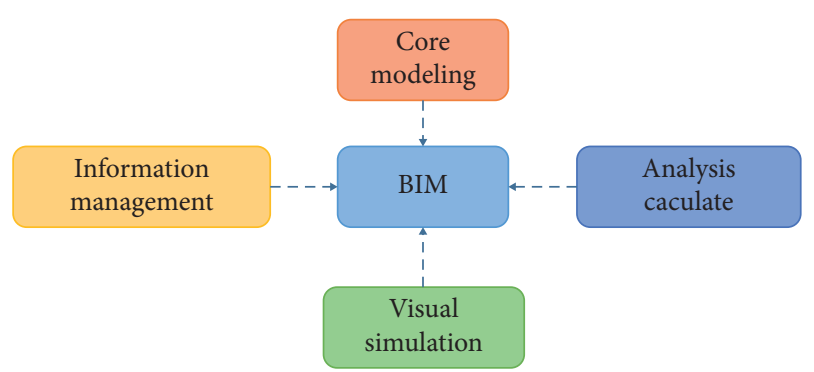

FIgURE 1: Classification diagram of the city information model.

$$
M_{e}=(Q+q)(X-r)
$$

where $q$ is the corresponding rated lifting weight when the amplitude is $R$; $Q$ is the weight of the spreader system; $M$ is the constant moment, which is generally the fixed value for the current tower crane; $X$ is the amplitude; and $R$ is the distance from the hinge point of the boom root to the rotation center, which is also known as the crane performance characteristic equation. In the reinforced block masonry building structure system, the wall mainly exists in four forms: I-shaped wall, L-shaped wall, T-shaped wall, and cross-shaped wall. Due to the problem of arrangement and combination, the arrangement order of blocks in the wall cannot be completely consistent, even if specific values are given. Therefore, each arrangement requires different requirements. If the use quantity of each block type is $M_{1}, M_{2}, M_{3}, \ldots, M_{j}, \ldots, M_{m}$, (2)must exist.

$$
N_{i}=M_{1}+M_{2}+M_{3}+\cdots+M_{m}
$$

The possible block combinations of this layer include all combinations of $N_{i}$. For the same type of blocks, the sequence of arrangement and combination is irrelevant and can be removed. Then, the final possible block arrangement number for the wall is as shown in formula (3):

$$
\frac{A_{N_{i}}^{N_{i}}}{A_{M_{1}}^{M_{1}} A_{M_{2}}^{M_{2}} A_{M_{3}}^{M_{3}}, \ldots, A_{M_{j}}^{M_{j}}, \ldots, A_{M_{m}}^{M_{m}}} .
$$

Therefore, by setting the necessary assumptions and limiting the type and order of block arrangement, we can uniquely determine the arrangement of wall blocks and then let the computer complete the massive but error-prone repetitive work of block arrangement.

\subsection{An Overview of Urban Wetland Parks and the Use of} Urban Information Models. The urban wetland park's landscape pattern is a kaleidoscope of different types, sizes, and shapes in space. Glacier formation, coastal lowland flooding, river erosion and accumulation, river otter and otter damming, and human activities are the main causes of wetland formation on a macroscale. The importance of an urban wetland park in a city not only contributes to the improvement of the city's ecological environment but also reflects the city's cultural connotation. It is a public gathering spot in the city that encourages residents to interact more. The urban wetland park definition model is shown in Figure 2.

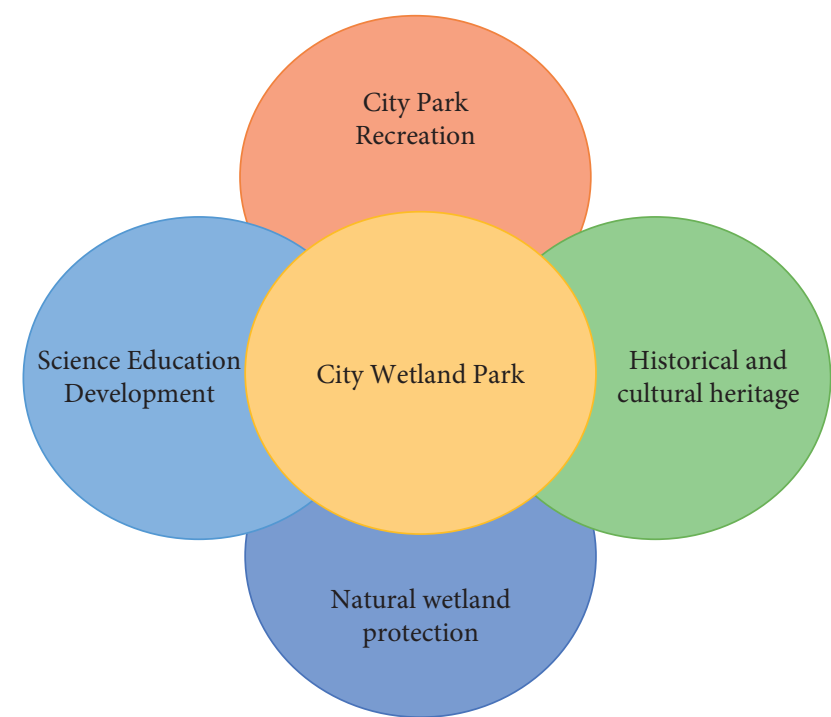

Figure 2: Definition map of the urban wetland park.

Reduce the interference and destruction of the natural wetland environment caused by the continuous expansion of the city, restore the original natural restoration ability of the wetland ecosystem, restore the diversity of wetland organisms, provide natural habitats for wildlife, improve the natural productivity of the wetland and the surrounding environment, and make the wetland system and the surrounding environment have the ability of self-renewal and restoration, which is what we are striving for now. The mechanism model of environmental ecological flow is shown in Figure 3.

What we have to do for this is as follows.

First, create a safe, comfortable, dynamic, and interactive urban wetland environment. The environment of organic urban wetland park includes protective units, productive units, harmonious units, and artificial units, with protective units as the main ones, and maintaining wetland resources means maintaining the ecological security of cities and regions. The wetland environment is maintained by the hands of the public through the implementation of some project activities, such as public welfare publicity, the occurrence of wild animals, tree adoption, and other activities, and the interactive development of human-nature-culture is formed.

Second, strengthen people's understanding of sustainable development. With the accelerating process of industrialization, people have questioned and discussed the traditional growth and development model under the pressure of ecological environment destruction, urban population growth, and economic growth. The core idea is to realize the coordinated development of society, environment, culture, economy, and resources. The most commonly used index system is as follows:

$$
H \times V \times O \times R
$$

where $H$ is the ecosystem health index, $V$ is the ecosystem vitality, $O$ is the ecosystem organization index, and $R$ is the ecosystem resilience. 


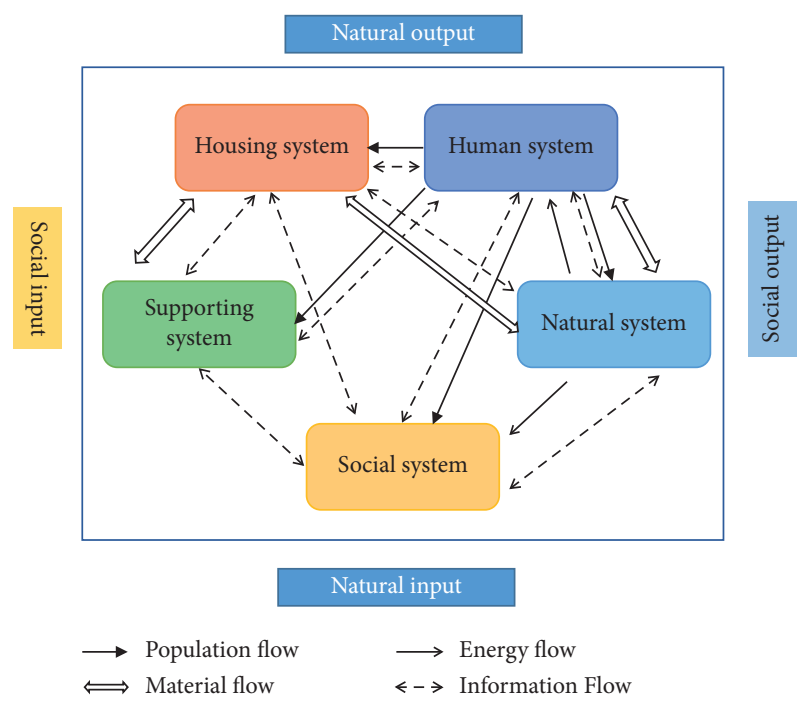

Figure 3: Model diagram of the mechanism of environmental ecological flow.

Third, it establishes control requirements for road, architectural, and human landscape construction. One of the prerequisites for the long-term development of urban wetlands is the establishment of reasonable tourist routes and the construction of reasonable roads. Controlling building height, density, and other indicators can help to reduce plane controller loss, coordinate with the environment, ensure visual perception, and impose some constraints based on the building shape. Traditional local characteristics should not be overlooked when creating an urban wetland model, and historical culture, humanistic artistic conceptions, and other factors can be properly integrated to promote the treasure and development of the urban wetland. The type of wetland quantity can be expressed by the following formula:

$$
S_{i j}=\left[\begin{array}{cccc}
S_{11} & S_{12} & \cdots & S_{1 n} \\
S_{21} & S_{22} & \cdots & S_{2 n} \\
\cdots & \cdots & \cdots & \cdots \\
S_{n 1} & S_{n 2} & \cdots & S_{n n}
\end{array}\right] .
$$

Fourth, when transforming urban wetland parks, we should also consider the regional environment and comprehensively analyze the data of topography, architecture, air, meteorology, and so on, such as air quality. People can arrange activities and time according to the air quality, which can reduce some unnecessary pollution and bring thoughtfulness to people who watch the scenery. AQI is calculated as follows:

$$
\begin{aligned}
\mathrm{AQI} & =\max \left\{\mathrm{IQAI}_{1}, \mathrm{IQAI}_{2}, \mathrm{IQAI}_{3}, \ldots, \mathrm{IQAI}_{n}\right\}, \\
\mathrm{IAQI}_{p} & =\frac{\mathrm{IAQI}_{\mathrm{Hi}}-\mathrm{IAQI}_{\mathrm{Lo}}}{\mathrm{BP}_{\mathrm{Hi}}-\mathrm{BP}_{\mathrm{Lo}}}\left(C_{p}-\mathrm{BP}_{\mathrm{Lo}}\right)+\mathrm{IAQI}_{\mathrm{Lo}} .
\end{aligned}
$$

Fifth, effective use of natural resources: in the whole process of building, we will consume a lot of resources, especially building materials, as well as waste gas generated in the whole process, which is very serious for the bad environment. To realize the rational use of the architectural landscape, realize the self-metabolism of the ecological environment, and reduce human pollution to nature, we should make effective use of the wind and solar lamps of natural buildings. Building materials should also be chosen for reuse in order to ensure material sustainability and reduce resource waste; there is also the discharged waste gas, which can be treated using special methods in order to achieve material recycling.

\section{Result Analysis and Discussion}

China's urban wetland park is a park that combines protection, science popularization, leisure, and other functions by rationally protecting and utilizing natural wetlands that are suitable for use as parks and incorporated into the city's green space system planning. From the perspective of regionality and culture, urban wetland park condenses the unique characteristics of the entire city. It is a city that exudes a distinct allure. Similar city business cards have evolved into an important tool for increasing urban competitiveness. The natural landscape of wetlands can provide visitors with a wealth of visual, physical, and mental stimulations. There are numerous urban wetland parks, each with its own unique characteristics. There are provincial urban wetland parks recognized by the provinces, as well as urban wetland parks in various regions that have not been declared, in addition to the national urban wetland parks recognized by the Ministry of Housing and Urban-Rural Development. The objects' complexity makes the research process extensive and challenging. Furthermore, each city's perception and focus on the urban wetland park vary, and wetland resources are distributed unevenly. The urban growth map of China is shown in Figure 4.

For the urban wetland park, SIM can also help the construction progress of the project road, which can be compiled according to the schedule, construction process, and construction scheme. The SIM can compare the collected actual progress data with the planned progress, clarify the deviation between the actual construction progress and the planned progress, and point out the direction for what adjustment measures to take, as shown in Figure 5.

In Figure 5, $\Delta T a$ is the time when the actual progress at $t_{a}$ time is ahead of the planned progress, and $\Delta Q_{\mathrm{a}}$ is the overcompleted workload at that time; $\Delta T \mathrm{~b}$ is the time when the progress lags behind at $t_{b}$ time, and $\Delta Q_{b}$ is the workload in arrears at that time. In addition, according to the progress information provided by the S-shaped curve of the actual progress, the time required for the unfinished work task can be predicted, the corresponding predicted progress curve can be made, and the predicted construction period of the project progress can be obtained. It can also be used to count the actual cost of completed work (BCWS), budget cost of completed work (BCWP), and budget cost of planned work (ACWP). The project progress can be measured by cost and budget, as shown in Figure 6.

Three basic parameters are used to obtain the progress deviation (SV) and cost deviation (CV) of the implementation of the plan, so as to judge the implementation of the plan and the project budget. 


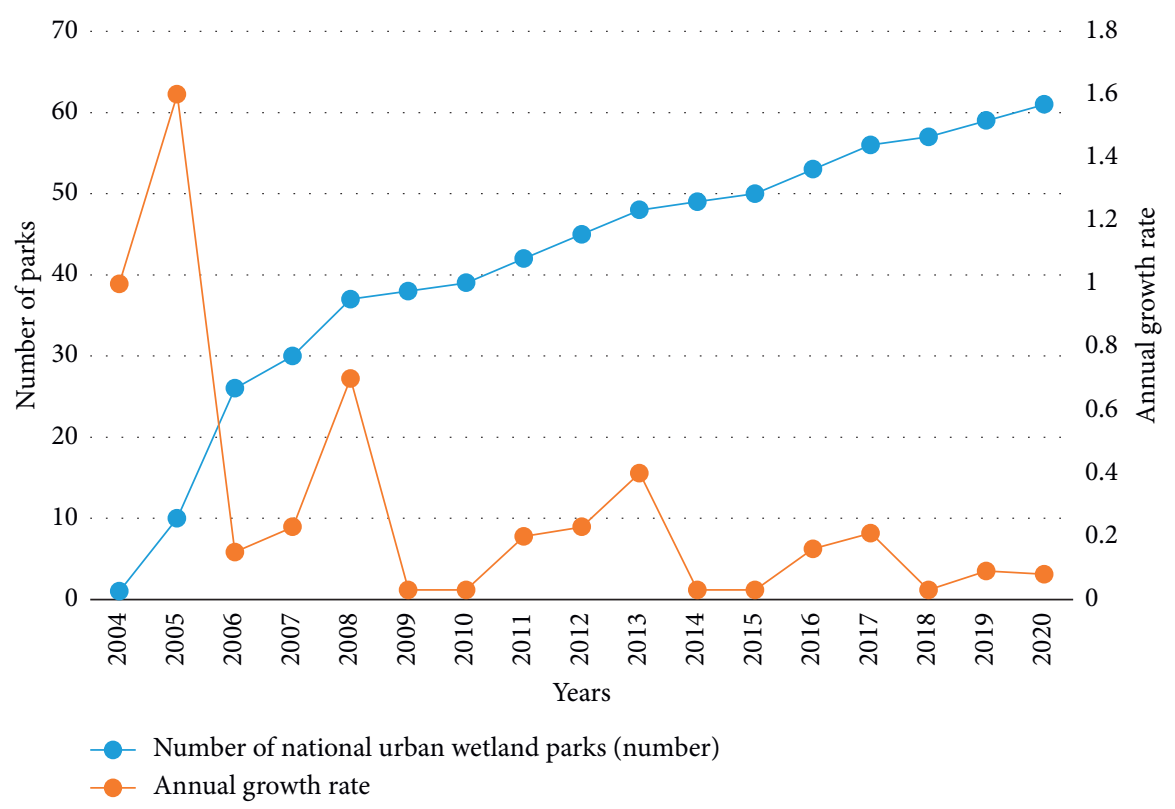

Figure 4: Distribution map of urban wetland parks in my country.

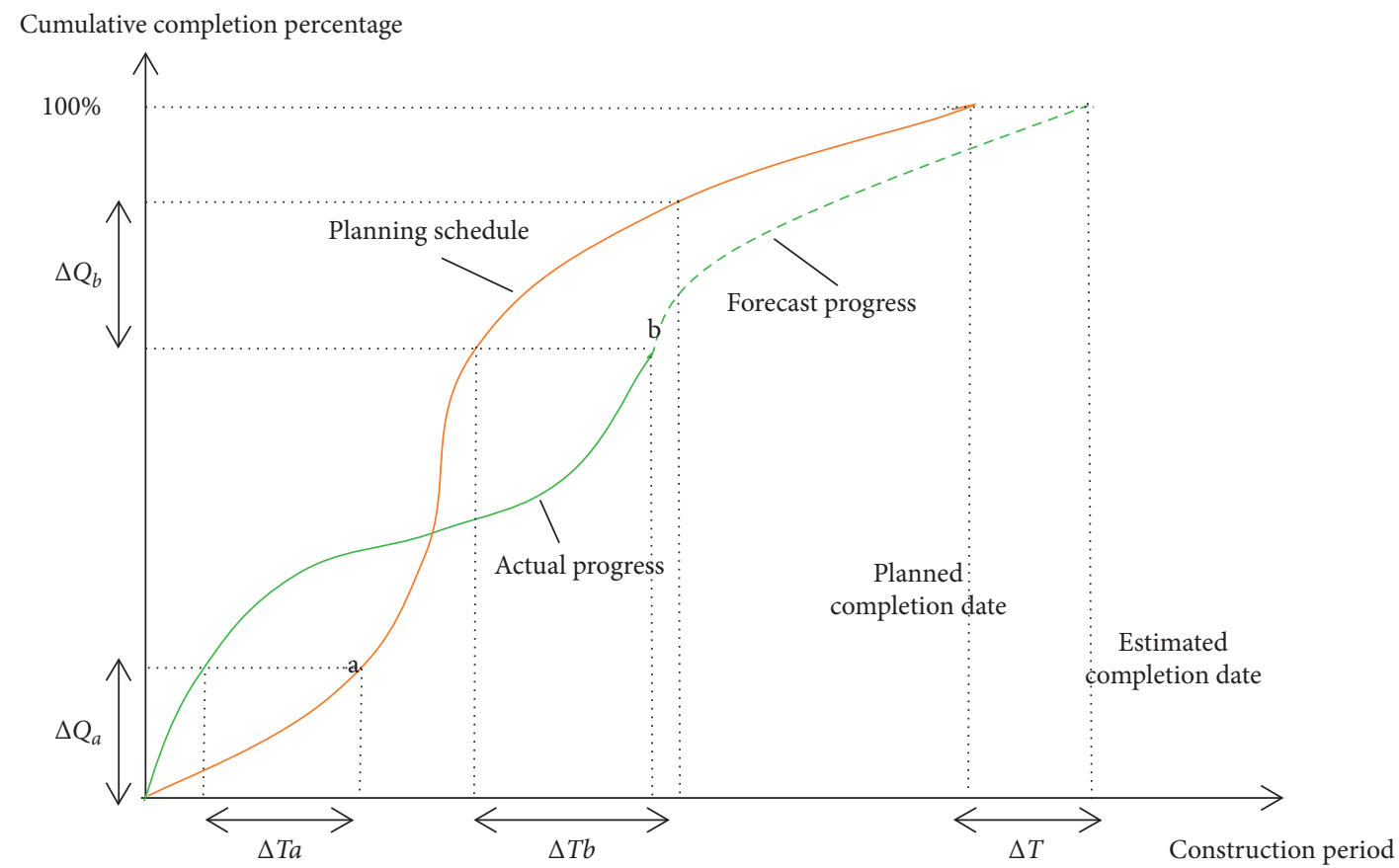

Figure 5: BIM time prediction chart.

Formulas (7) and (8) and five algorithms, actual value, CKUNR, AIQ, BP, and Elm, can be used synchronously. Based on the accuracy of the BIM, the air environment around our urban wetland buildings has been tested. Using these algorithms, the pollution in the air and the predictability of the air environment can be well tested, providing benefits for sustainable development and promoting the development of the ecological environment, as shown in Figure 7.

This paper also studies the information of landscape patches from PD and AI indices and analyzes the sensitivity of viewing index to scale. The map also includes rural areas, urban fringe areas, and built-up areas. The landscape types are complete and representative. According to the equidistant intervals, the PD and AI values of different sizes corresponding to the center line of the transect are extracted. At $2250 \mathrm{~m}$, the change continuity of the landscape index with sampling points in the transect is good, and the change curve is gentle. When the analysis range is greater than or less than $2250 \mathrm{~m}$, the change of the landscape index fluctuates greatly, as shown in Figures 8 and 9.

BIM technology can simply provide an information sharing platform that connects all project participants and 


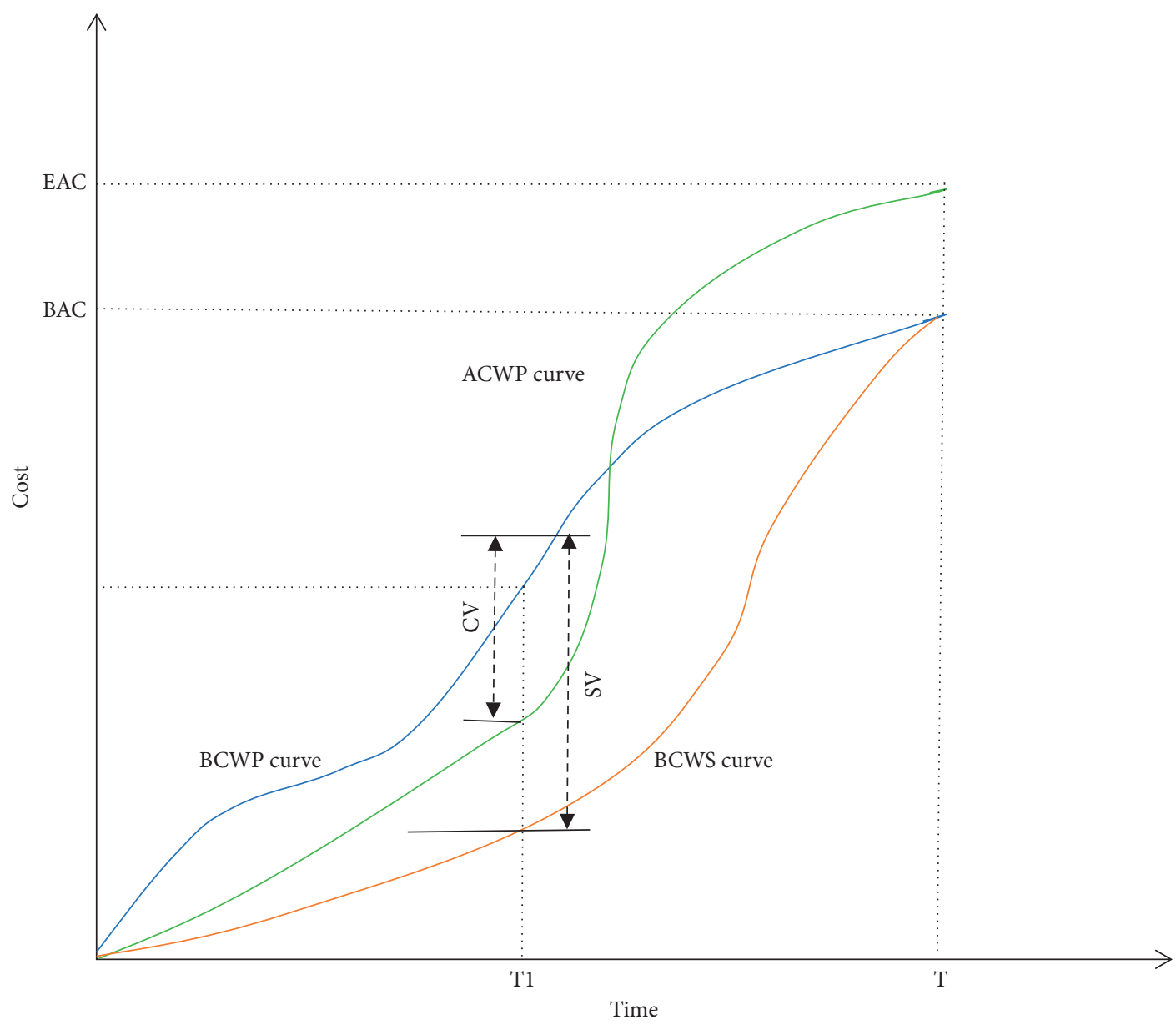

Figure 6: Cost engineering forecast.

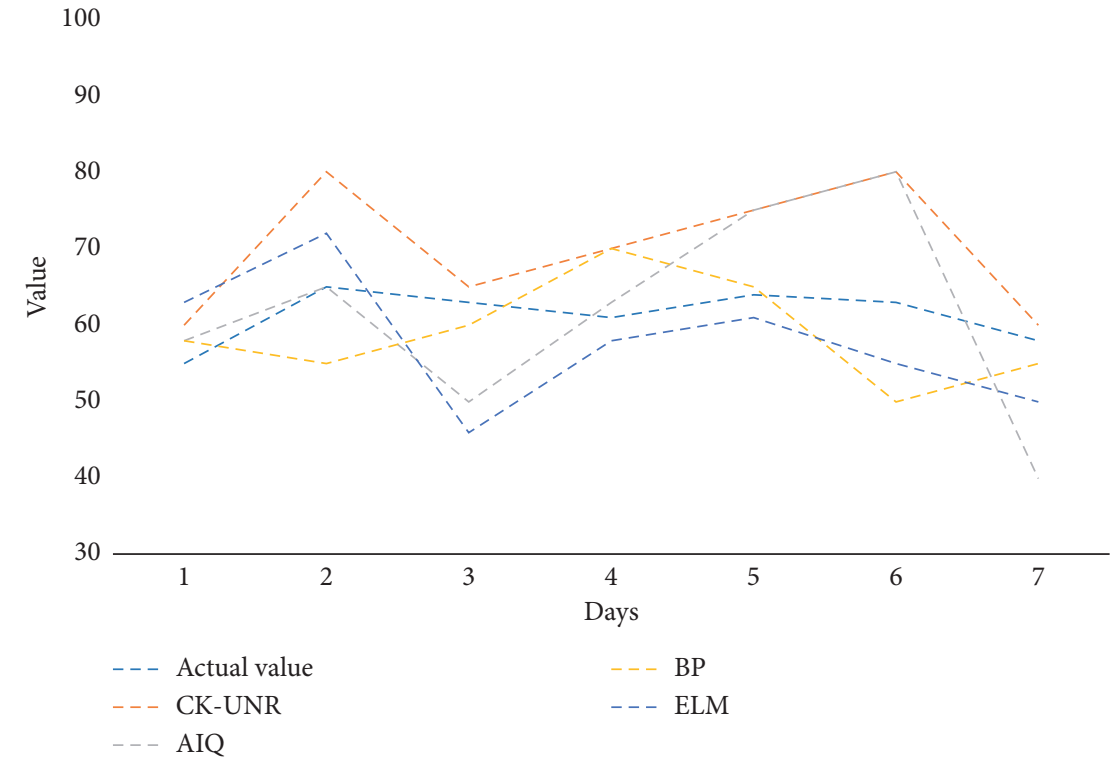

FIgURE 7: One week of air environment test.

runs throughout the construction process. As a result, in the early stages of the project, it is possible to ensure the rationality of the project cost estimation, budgetary estimate, and budget, as well as the effective transmission of cost management information between stages. The project participants can simulate the project's construction process, 


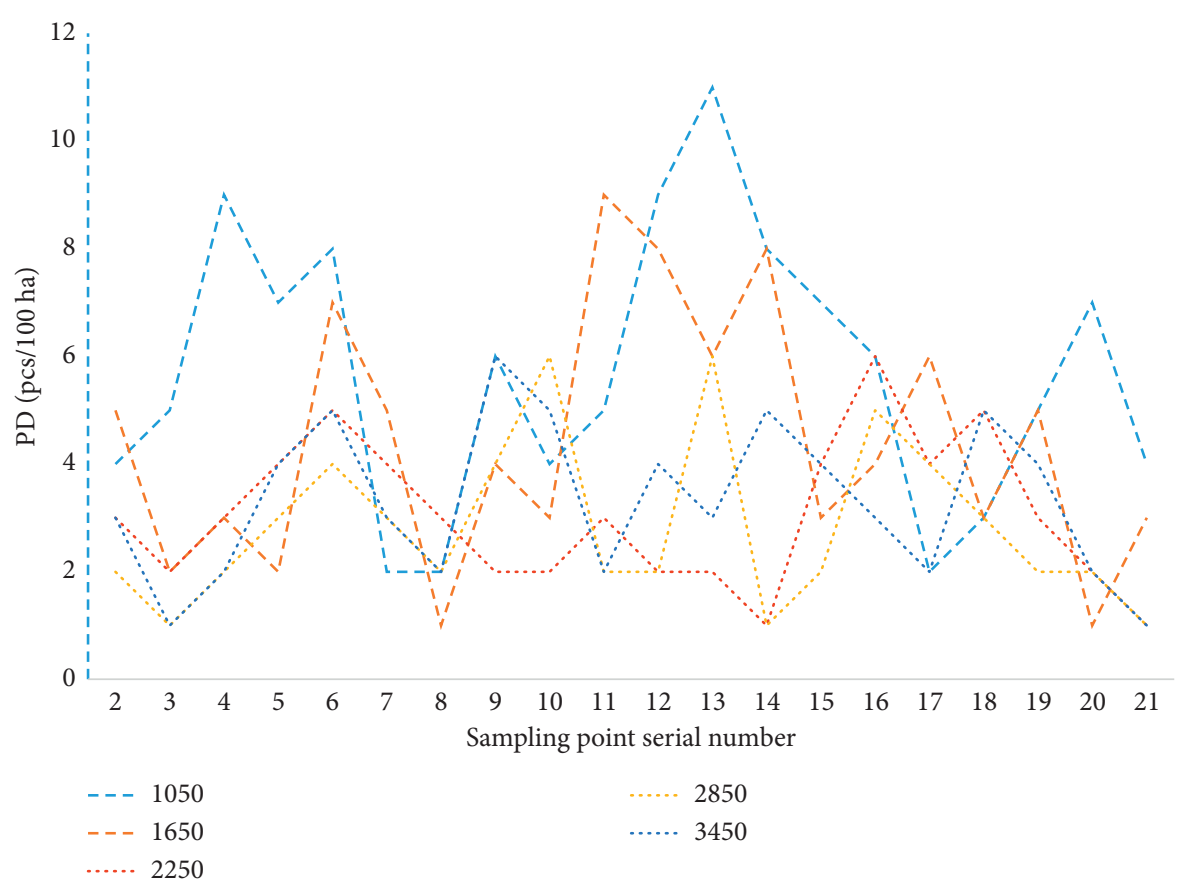

FIgURe 8: Landscape patch test.

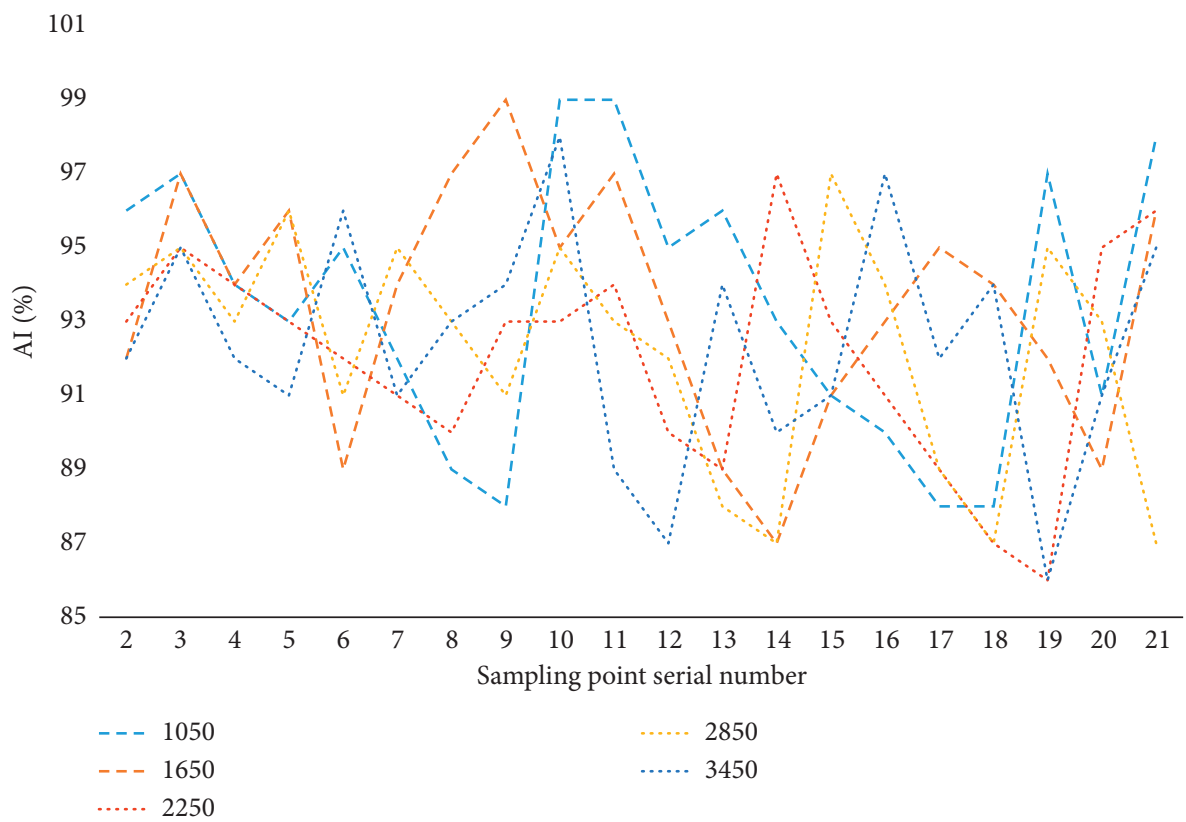

FIGURE 9: Aggregation index graph.

dynamically compare the project's actual cost to the target value, and achieve dynamic real-time monitoring of the project cost using BIM visualization and simulation. Compare the unforeseen fees that must be retained for traditional construction projects with the accuracy calculated by the BIM using formula (1), as shown in Figure 10.

From the above test statistics, it can be seen that BIM can bring us great portability, provide accurate data support, and not only greatly change the time but also make a good plan for the whole urban wetland building with its accuracy. We can plan the errors calculated by the BIM in advance and prepare the plan in advance. This shows the importance of the BIM to urban development.

\section{Conclusions}

Today and in the future, urbanization is a worldwide phenomenon. One of the most prominent characteristics of 


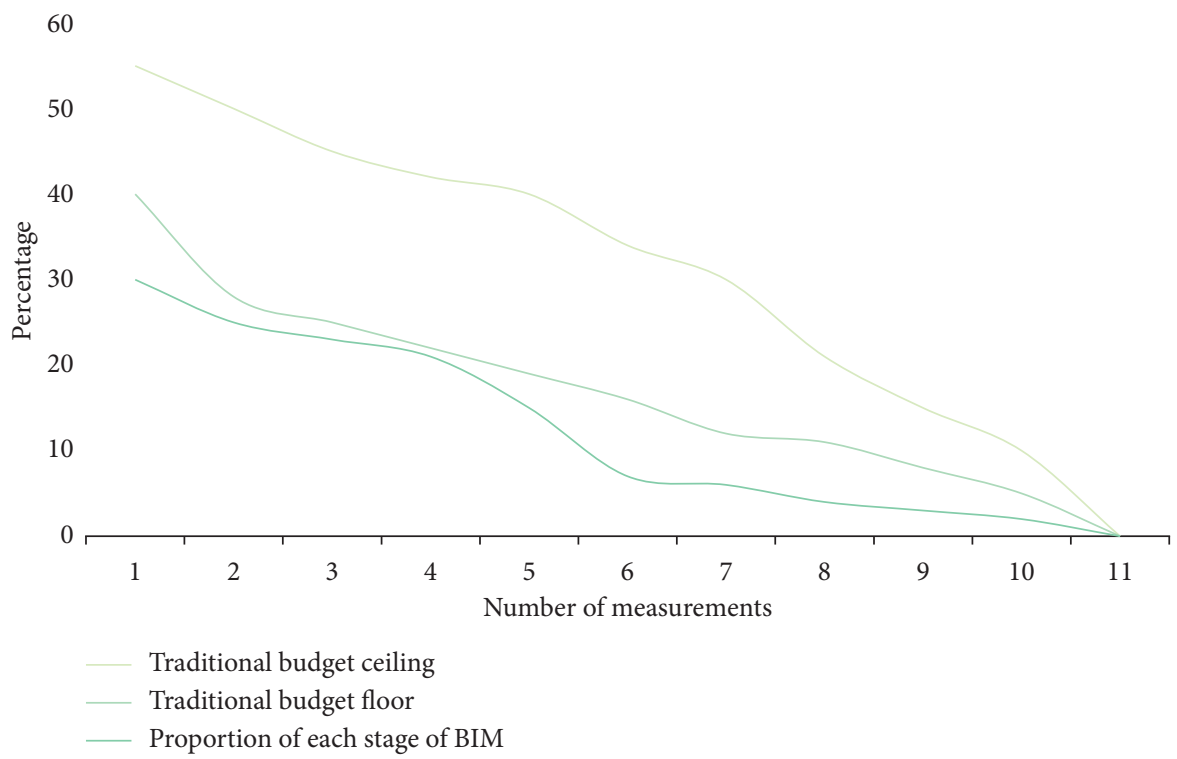

FIGURE 10: Comparison of the unforeseen costs that need to be retained for projects with traditional construction methods and the accuracy calculated by the BIM company.

urbanization is the expansion of space. Urbanization has drastically altered the natural landscape's type and pattern, interfering with natural ecological processes and obstructing material and energy flow. The creation of the landscape for urban wetland park is a complex project that incorporates system theory and methodology. It is difficult to discuss all of the factors from a single perspective. The urban wetland park's wetland subject is ecologically sensitive. Its planning and design must take into account the topography, climate, hydrological conditions, history, and culture of the site. China's urban planning is still being investigated. It primarily respects the natural environment and regional characteristics, properly addresses the relationship between the landscape architecture and the environment, locates the functional nature of the urban wetland park in a reasonable location, moderately constructs artificial facilities, and chooses construction technology in a reasonable manner. Urban wetland parks have a significant functional value in urban construction and development, which is primarily reflected in the value of ecological protection, cultural communication, and economic development. BIM will allow construction to move forward, reduce errors, and increase profits. The widespread use of the BIM can help the future construction site move toward modernization, industrialization, standardization, visualization, and intelligence. To improve the cultural landscape construction level of urban wetland park in all aspects, we must begin by infiltrating the city's local characteristic culture, implementing the humanistic concept, and constructing diversified financing channels. This paper investigates the BIM of an urban wetland park and proposes architectural planning to make the urban wetland more dynamic and ecologically sustainable. Of course, the city will work to improve the quality of the environment and promote man-nature harmony.

\section{Data Availability}

The data used to support the findings of this study are included within the article.

\section{Conflicts of Interest}

The author declares that there are no conflicts of interest.

\section{Acknowledgments}

This study was supported by the Fund Project Guangdong University of Science and Technology "Innovation and School Strengthening Project" (research)—Guangdong University of Science and Technology "Elastic" Landscape Research Institute (Project no. GKY-2019CQYJ-6).

\section{References}

[1] Ye Wang, "Research on plant landscape construction technology and construction strategy of urban wetland park-taking huzhou city wetland park landscape construction as an example," $A b$ stracts of Chinese Horticulture, vol. 32, no. 4, p. 3, 2016.

[2] S. Wang, "Research on landscape design in wetland park construction," Smart City, vol. 12, no. 12, p. 1, 2016.

[3] L. Yan, "The application of humanized landscape design in urban theme parks-Taking Liuzhou City Wetland Park as an example," Modern Horticulture, vol. 10, no. 10, p. 2, 2018.

[4] L. Liu and Y. Hu, "The application of BIM in the teaching of "landscape architecture and architectural design," China Forestry Education, vol. 5, no. 5, p. 4, 2016.

[5] Lu Jin, "Research on landscape architecture of urban wetland park," Architecture and Decoration, vol. 7, no. 7, p. 2, 2017.

[6] S. Song, C. Albert, and M. Prominski, "Exploring integrated design guidelines for urban wetland parks in China," Urban 
Forestry and Urban Greening, vol. 53, Article ID 126712, 2020.

[7] Y. Lei, "Ecological landscape design of yunnan urban wetland park," Architectural Engineering Technology and Design, vol. 000, no. 012, p. 5917, 2017.

[8] L. Zhou, "Analysis of the British BIM (BIM) strategy and its implementation in the landscape architecture industry," Landscape Architecture, vol. 4, no. 4, p. 7, 2016.

[9] Z. Xie and Y. Lu, "Research on the landscape design of urban ecological wetland park," Jiangxi Building Materials, vol. 7, no. 7 , p. 2, 2016.

[10] Z. Lu, R. Cui, C. Shen, and M. Zhao, "Planning and design practice of wetland park based on the theory of landscape ecology: a case study of Wangjiatan Wetland Park in Changyuan City," Acta Ecologica Sinica, vol. 40, no. 22, p. 9, 2020.

[11] W. Luan, X. Tian, and X. Tiguli Azati, "Research on LIM digital platform based on landscape architecture," Modern Horticulture, vol. 7, no. 22, p. 4, 2018.

[12] W. Wang, J. S. Chen, L. Fan, and J. Lu, "Tourist experience and wetland parks: a case of Zhejiang, China," Annals of Tourism Research, vol. 39, no. 4, pp. 1763-1778, 2012.

[13] Bloomberg, "Analysis of BIMBIM visual roaming presentation analysis technology," Value Engineering, vol. 37, no. 34, p. 2, 2018.

[14] Yi Zheng, "Research and analysis on the landscape design of urban wetland parks," Architectural Engineering Technology and Design, vol. 000, no. 014, Article ID 3163, 2016.

[15] S. He, "Research on the landscape construction of urban wetland park," Urbanism and Architecture, vol. 26, no. 26, p. 1, 2016.

[16] X. Hu, Research on the Landscape Design of Urban Ecological Wetland Park Engineering Technology Architecture, 5, Article ID 00126, Abstract Edition, 2016.

[17] Y. Cao and S. Shen, "Urban wetland park landscape architecture," Architecture and Culture, vol. 9, no. 9, p. 2, 2018.

[18] S. Miao and X. Xu, "Research on the landscape design of urban wetland park based on ecological concepts-take the landscape engineering design of binhe south road in yongjing county as an example," Beauty and Times: City, vol. 8, no. 9, p. 2, 2016.

[19] G. Yu and P. Ren, "Architectural design management mode based on BIM (BIM)," Architectural Engineering Technology and Design, vol. 000, no. 015, p. 2380, 2016.

[20] J. Huang, "The application of BIM in green buildings," Journal of Neijiang Normal University, vol. 32, no. 2, p. 3, 2017.

[21] T. Xu, "BIM construction in construction drawing design of building structure," Building Technology Development, vol. 44, no. 4, p. 2, 2017.

[22] Z. Zhang, "Analyze the plant configuration in garden landscape design from the ecological point of view-Taking urban wetland park as an example," Urbanism and Architecture, no. 33, p. 1, 2016.

[23] X. Sun, "Research on the landscape design of constructed wetland-taking dongying forest wetland park as an example," Modern Horticulture, vol. 44, no. 6, p. 2, 2021.

[24] L. Xing, "Overview of BIM (BIM)," Science and Technology Innovation, vol. 20, no. 20, p. 2, 2020.

[25] X. Dai and Y. Xu, "Landscape application analysis of aquatic plants in urban wetland parks," China Wild Plant Resources, vol. 39, no. 10, p. 5, 2020.

[26] J. Kong, H. Wang, X. Wang, X. Jin, X. Fang, and S. Lin, "Multi-stream hybrid architecture based on cross-level fusion strategy for fine-grained crop species recognition in precision agriculture," Computers and Electronics in Agriculture, vol. 185, Article ID 106134, 2021.
[27] X.-B. Jin, W.-Z. Zheng, J.-L. Kong et al., "Deep-learning temporal predictor via bidirectional self-attentive encoder-decoder framework for IOT-based environmental sensing in intelligent greenhouse," Agriculture, vol. 11, no. 8, 802 pages, 2021.

[28] J. Zeng, "Analysis on the landscape design of urban wetland park," Anhui Architecture, vol. 26, no. 10, 2 pages, 2019.

[29] Y. Niu, "The application of landscape design in the construction of urban wetland parks," Modern Horticulture, no. 4, 2 pages, 2019.

[30] C. Jin, M. Xu, and X. Zhou, "Classification method of architectural space function based on BIM (BIM)," Computer Applications and Software, vol. 35, no. 11, 6 pages, 2018. 